\title{
Polimorfismo en el gen COMT en una muestra de gestantes normales y con restricción del crecimiento intrauterino en un hospital de Lima
}

\author{
COMT gene polymorphism in a sample of pregnant women with \\ intruterine growth restriction in a Lima hospital
}

\author{
José Pacheco-Romero ${ }^{1}$, Doris Huerta ${ }^{2}$, Oscar Acosta ${ }^{2}$, Santiago Cabrera ${ }^{3}$ \\ ${ }^{1}$ Instituto de Investigaciones Clínicas, Facultad de Medicina, Universidad Nacional Mayor de San Marcos. \\ ${ }^{2}$ Centro de Investigación de Bioquímica y Nutrición, Facultad de Medicina, Universidad Nacional Mayor de San Marcos. \\ ${ }^{3}$ Hospital Materno Infantil San Bartolomé, Lima, Perú.
}

\begin{abstract}
Resumen
Antecedentes: Los procesos fisiopatológicos que ocurren a nivel celular y molecular en la restricción de crecimiento intrauterino (RCIU) son aún desconocidos. La catecol-O-metiltransferasa (COMT) es una enzima de fase II que inactiva los catecol estrógenos al transferir un grupo metílico. Se conoce un polimorfismo funcional Val158 Met en el gen COMT como un marcador susceptible para diversas enfermedades maternoperinatales, existiendo estudios que sugieren que el alelo que codifica una COMT de baja actividad puede ser un marcador susceptible para RCIU. Por lo tanto, el estudio del polimorfismo COMT ofrece una nueva estrategia para la evaluación de marcadores genéticos que pueden ser utilizados para la detección de ciertas alteraciones asociadas al embarazo. Objetivos: Establecer la asociación entre el polimorfismo Val158Met catecol-0-metiltransferasa (COMT) y la restricción de crecimiento intrauterino. Institución: Facultad de Medicina, Universidad Nacional Mayor de San Marcos, Lima, Perú. Diseño: Estudio tipo relacional (asociativo), con diseño observacional, tipo caso-control (no experimental). Materiales: Muestra de sangre materna de parturientas. Métodos: Durante el año 2011, se obtuvo 81 muestras para genotipaje del gen COMT. De ellas, 26 (32,1\%) correspondieron a parturientas con RCIU (casos) y 55 (67,9\%) a muestras de madres de hijos sin RCIU (controles). La distribución de los genotipos fue evaluada usando la prueba de chi cuadrado. Se comprobó la distribución proporcional de los genotipos en los grupos con RCIU y sin RCIU con la hipótesis nula de Hardy-Weinberg. Las madres participantes firmaron un consentimiento informado. Principales medidas de resultados: Asociación entre los genotipos COMT y la RCIU, y entre los alelos COMT Val/Met y la RCIU. Resultados: Las distribuciones de los genotipos en los grupos con RCIU y sin RCIU estuvieron de acuerdo a la hipótesis nula de Hardy-Weinberg. Al relacionar los genotipos COMT Val/Met con la condición de $\mathrm{RCIU}$, la prueba $\mathrm{X}^{2}=1.8057, \mathrm{gl}=2, \mathrm{p}=0.4054$, no encontró asociación entre dichos genotipos y la RCIU. Al determinar la asociación entre los alelos COMT y la RCIU, tampoco se encontró asociación entre los alelos COMT Val/Met y la condición de RCIU en la muestra estudiada, con prueba $X^{2}=0.3659, g l=1, p=0.5453$. Conclusiones: No se encontró asociación entre los genotipos COMT y la RCIU, ni entre los alelos COMT Val/Met y la RCIU, en la muestra estudiada.
\end{abstract}

Palabras clave: Restricción de crecimiento intrauterino, fisiopatología, catecol-O-metiltransferasa, catecol estrógenos, polimorfismo Val158Met del gen COMT.

\section{Abstract}

Background: Cellular and molecular events in the pathophysiology of intrauterine growth restriction (IUGR) are still unknown. CathecolO-methyltransferase (COMT) is a phase II enzyme that inactivates cathecol estrogens by transferring a methyl group. A functional polymorphism Val158 Met in COMT gene is known as susceptible marker for diverse maternal and perinatal diseases, and studies suggest the allele codifying a low activity COMT may be a susceptible marker for IUGR. COMT polymorphism study may be a new strategy to determine genetic markers that might be used for detection of certain disorders related to pregnancy. Objectives: To determine association of Val158Met cathecol-O-methyltransferase (COMT) polymorphism and intrauterine growth restriction. Setting: Faculty of Medicine, Universidad Nacional Mayor de San Marcos, Lima, Peru. Design: Relational (associative), observational, casecontrol (non-experimental) study. Materials: Maternal blood samples obtained following delivery. Methods: During 2011, 81 blood samples were obtained from post partum mothers for COMT gene genotyping, 26 (32.1\%) were mothers with IUGR (cases) and 55 $(67.9 \%)$ without IUGR (controls). Genotype distribution was determined by chi square test. Genotypes proportional distribution in IUGR and non-IUGR groups was determined with Hardy-Weinberg's null hypothesis. All women signed informed consent. Main outcome measures: Association of COMT genotypes and IUGR, and between COMT Val/Met alleles and IUGR. Results: Genotype distribution in IUGR and non-IUGR groups agreed with Hardy-Weinberg null hypothesis. There was no association of COMT Val/Met genotypes and IUGR, $X^{2}=1.8057, g \mid=2, p=0.4054$. There was no association between COMT Val/Met alleles and IUGR, $X^{2}=0.3659, g \mid=1, p=0.5453$. Conclusions: No association was found either between COMT genotypes and IUGR or between COMT Val/Met alleles and IUGR.

Key words: Intrauterine growth restriction, pathophysiology, cathecol-O-methyltransferase, cathecol estrogens, COMT gene Val158Met polymorphism.

An Fac med. 2013;74(2):129-32 


\section{INTRODUCCIÓN}

La restricción de crecimiento intrauterino (RCIU) tiene una frecuencia de 10 a $15 \%$ en la población general, con impacto importante en la morbilidad de la población materna y morbimortalidad pediátrica ${ }^{(1)}$. En trabajos previos sobre preeclampsia/eclampsia (PE/E) en la mujer peruana, se ha encontrado que en la población sin $\mathrm{PE} / \mathrm{E}$ la incidencia de peso pequeño al nacer ocurrió en $7,6 \%$ de los recién nacidos. Mientras tanto, en la preeclampsia fue $18 \%$ y en la eclampsia 23,6\%, con significación estadística ${ }^{(2)}$.

Los fetos y recién nacidos con RCIU poseen mayor riesgo de morbimortalidad perinatal y neonatal, así como de secuelas neurológicas ${ }^{(3)}$, lo que ha sido corroborado en nuestro medio ${ }^{(1,4,5)}$. Por otra parte, existe evidencia epidemiológica de asociación de RCIU con hipertensión arterial, enfermedad coronaria y diabetes tipo II -en general con el síndrome metabólico - en la etapa adulta ${ }^{(6-8)}$, lo cual representa un problema de salud pública y fuerte impacto en discapacidad, carga de enfermedades metabólicas y relacionadas y años de vida saludables ${ }^{(9,10)}$.

No existe manera de predecir tempranamente la ocurrencia de la RCIU -salvo con la ultrasonografía, pero haciendo el diagnóstico generalmente tardío en el embarazo ${ }^{(11,12)}$ - o de entidades relacionadas, como la $\mathrm{PE} / \mathrm{E}^{(13)}$.

Los procesos fisiopatológicos que ocurren a niveles celular y molecular en la RCIU son aún desconocidos. La catecol-O-metiltransferasa (COMT) es una enzima fase II que inactiva los catecol estrógenos al transferir un grupo metílico ${ }^{(6,14,15)}$. Se conoce al polimorfismo funcional Val158Met en el gen COMT como un marcador susceptible para diversos tipos de enfermedades materno perinatales, entre ellas la RCIU ${ }^{(16)}$.

Existen estudios que sugieren que el alelo que codifica un COMT de actividad baja (COMT-L) puede ser un marcador susceptible para RCIU ${ }^{(6)}$. Por lo tanto, el estudio del polimorfismo de
COMT ofrece nuevas estrategias para la evaluación de novedosos marcadores genéticos en gestantes, que pueden ser utilizados para la detección de ciertas alteraciones asociadas al embarazo. Por otro lado, el polimorfismo COMT-L ha sido estudiado en gestantes con RCIU y los resultados sugieren una probable asociación de este polimorfismo con RCIU, específicamente el alelo de baja actividad ${ }^{(6)}$. Por lo tanto, el análisis de este polimorfismo puede permitir generar estrategias dirigidas a la prevención, tamizaje, diagnóstico y manejo de la RCIU.

El objetivo general del presente estudio fue establecer la asociación entre el polimorfismo Val158Met catecol-Ometiltransferasa (COMT) y la restricción de crecimiento intrauterino, siendo los objetivos específicos determinar las frecuencias genotípicas y alélicas del polimorfismo de COMT en gestantes con RCIU y embarazos normales, así como la asociación del polimorfismo COMT en gestantes que cursaban con RCIU (casos) y con embarazos normales (controles).

Tuvimos, además, en consideración que el presente estudio en nuestro medio fortalecerá el estudio de la COMT y otros marcadores genéticos en condiciones fisiológicas y patológicas del embarazo, pues los resultados podrán incentivar al desarrollo de nuevos estudios que contribuyan a la comprensión de la fisiopatología y la genética de la RCIU.

\section{MÉTODOS}

El presente estudio fue una investigación tipo relacional (asociativa), con diseño observacional, tipo caso-control (no experimental). La hipótesis planteada fue que existe asociación entre el polimorfismo del gen de la COMT y la RCIU en mujeres embarazadas.

La población en estudio estuvo compuesta por gestantes residentes en Lima. La muestra seleccionada fue de gestantes mayores de 18 años atendidas en el Hospital Nacional Docente Madre-Niño San Bartolomé, durante el año 2011, distribuidas en grupos de gestantes con RCIU y gestantes con embarazo normal. Los criterios de inclusión para los casos fueron de gestantes con diagnóstico de RCIU, considerado como un peso al nacer por debajo del 10 percentil, de acuerdo a la tabla de Lubchenco. Los criterios de exclusión para los casos fueron los recién nacidos con malformaciones congénitas o que pertenecieran a embarazos gemelares. Los criterios de inclusión para los controles fueron gestantes con recién nacidos sin RCIU, excluyéndose los embarazos múltiples y/o fetos muertos.

La recolección de datos fue realizada con una ficha ad hoc elaborada para los datos personales y clínicos de la madre y el neonato. Las madres que participaron firmaron un consentimiento informado, que incluía la finalidad de la investigación, las condiciones en que se realizaba, procedimientos, riesgos y beneficios, confidencialidad de los resultados y los datos de los investigadores. Se consideró las pautas mencionadasen la Declaración de Helsinki ${ }^{(17)}$ y se contó con la aprobación del Comité de Ética del Hospital.

Las muestras fueron obtenidas al momento del parto de madres con diagnóstico prenatal y/o confirmación posnatal de recién nacido con RCIU. Se extrajo $3 \mathrm{~mL}$ de sangre venosa en tubos Vacutainer que contenían anticoagulante, la que fue trasladada al Centro de Investigación de Bioquímica y $\mathrm{Nu}$ trición, para su procesamiento.

El genotipaje del polimorfismo VAL/ MET de la COMT fue realizado en el Centro de Investigación de Bioquímica y Nutrición (CIBN) de la Facultad de Medicina de la UNMSM, en el laboratorio de Ácidos Nucleicos. Para ello, se procedió a purificar el ADN, utilizando un kit comercial. El polimorfismo del gen COMT fue determinado por la técnica del PCR/RFLP, estandarizado en nuestro laboratorio (CIBN) con primers específicos, y las variantes genotípicas fueron obtenidas por digestión con la enzima de restricción NlaIII. Para conocer la calidad del ADN y la visua- 
Tabla 1. Genotipos COMT Val/Met y condición restricción de crecimiento intrauterino (RCIU).

\begin{tabular}{cccc} 
Genotipos COMT & RCIU & Sin RCIU & Total \\
\hline Met/Met & 3 & 8 & 11 \\
Val/Met & 15 & 23 & 38 \\
Val/Nal & 8 & 24 & 32 \\
Total & 26 & 55 & 81 \\
\hline
\end{tabular}

Prueba $X^{2}=1.8057, g l=2, p=0.4054$ (no significativo)

lización de los amplificados se utilizó electroforesis en gel de agarosa al $2 \%$, siendo teñidos con bromuro de etidio, y los digeridos fueron visualizados en geles de poliacrilamida teñidos con nitrato de plata.

La distribución de los genotipos fue evaluada usando la prueba de chi cuadrado. Se comprobó la distribución proporcional de los genotipos en los grupos con RCIU y sin RCIU con la hipótesis nula del equilibrio de Hardy-Weinberg.

\section{RESULTADOS}

Se obtuvo 81 muestras para genotipaje del gen COMT. De ellas, $26(32,1 \%)$ correspondieron a parturientas con hijos diagnosticados de RCIU (casos) y 55 (67,9\%) fueron muestras de madres de hijos sin RCIU (controles).

Se comprobó si las distribuciones de los genotipos en los grupos con RCIU y sin RCIU eran proporcionales, determinándose que estaban de acuerdo a la hipótesis nula del equilibrio de HardyWeinberg.

$\mathrm{Al}$ relacionar los genotipos COMT $\mathrm{val} / \mathrm{met}$ con la condición de RCIU (tabla 1), la prueba $X^{2}=1,8057, \mathrm{gl}=2$, $\mathrm{p}=0,4054$ señaló no encontrar asociación entre dichos genotipos y la RCIU, para la muestra analizada.

Tabla 2. Alelos COMT Val/Met y condición restricción de crecimiento intrauterino (RCIU).

\begin{tabular}{ccc} 
Alelos COMT & RCIU & Sin RCIU \\
Met & 21 & 39 \\
Val & 31 & 71 \\
Total & 52 & 110 \\
\hline
\end{tabular}

Prueba $X^{2}=0.3659, g l=1, p=0.5453$ (no significativo).
Al determinar la asociación entre los alelos COMT y la RCIU (tabla 2), tampoco se encontró asociación entre los alelos COMT Val/Met y la condición de RCIU en la muestra estudiada, con prueba $\mathrm{X}^{2}=0,3659, \mathrm{gl}=1, \mathrm{p}=0,5453$, no significativa.

\section{DISCUSIÓN}

La COMT es una enzima clave en la degradación de catecolaminas y estrógenos. Se ha descubierto variantes de COMT de actividad alta y baja, debido a modificaciones de base única. Un polimorfismo con implicancias funcionales es un cambio de base $G$ por $A$, el polimorfismo COMT Val108/158Met. Este polimorfismo resulta en la sustitución del aminoácido valina por metionina en los codones 108 y 158, en las isoformas de COMT soluble y ligada a la membrana, respectivamente. El alelo Met(A) de este polimorfismo se asocia con una disminución de 3 a 4 veces de la actividad de la enzima COMT y varias condiciones clínicas, como la percepción del dolor, trastornos psiquiátricos, hipertensión y enfermedad cardiaca ${ }^{(18,19)}$. Los metabolitos 4- y 6-hidroxilados de estrógenos han sido relacionados a carcinogénesis -como de mama, endometrio, entre otros, mientras que sus metabolitos 2-hidroxilados han mostrado efectos antiangiogénicos ${ }^{(20,21)}$.

En la placenta, la aromatasa P-450 funciona como 2-hidroxilasa de estrógeno, así como 19-, 1 beta- y 2-hidroxilasa de andrógeno. Por ello, la aromatasa placentaria es responsable de los niveles muy elevados de catecolestrógeno y 19-hidroxiandrógeno en la gestación ${ }^{(22)}$.
Estudios recientes sugieren que la deficiencia ${ }^{(23)}$ y el polimorfismo genético de catecol-O-metiltransferasa (COMT) se asocian con la preeclampsia ${ }^{(24-26)}$. Habíamos señalado en la introducción que se conoce un polimorfismo funcional Val158Met en el gen COMT como un marcador susceptible para diversos tipos de enfermedades materno perinatales, entre ellas la RCIU ${ }^{(15,24)}$. Asimismo, que existen estudios que sugieren que el alelo que codifica un COMT de actividad baja (COMT-L) puede ser un marcador susceptible para RCIU, específicamente el alelo de actividad baja ${ }^{(6)}$. Por lo tanto, el estudio del polimorfismo de COMT podría ser una estrategia para la evaluación de nuevos marcadores genéticos en gestantes, de manera de detectar ciertas alteraciones asociadas al embarazo.

En la investigación que hemos realizado para determinar si existía asociación entre el polimorfismo del gen de la COMT y la RCIU en mujeres embarazadas en una muestra de Lima, Perú, no se ha hallado asociación entre los genotipos COMT y la RCIU, ni entre los alelos COMT val/met y la RCIU. Nuestros resultados no confirman las apreciaciones anteriores de que el gen COMT es un marcador susceptible para diversos tipos de enfermedades materno perinatales, entre ellas la RCIU ${ }^{(15)}$, y que el alelo que codifica un COMT de actividad baja (COMT-L) podía ser un marcador susceptible para RCIU ${ }^{(6)}$.

Existen estudios en la literatura en los que no se ha hallado asociación de la expresión del COMT y la preeclampsia ${ }^{(27)}$. Sin embargo, la esperanza de dicha asociación es observada en nuevos estudios, como el de rescate experimental con sildenafil de fetos con RCIU (28).

Con relación a nuestros hallazgos, estos podrían deberse principalmente al tamaño pequeño de la muestra o a otros factores no determinados.

En conclusión, no hemos hallado asociación entre los genotipos COMT y la RCIU, ni entre los alelos COMT val/ met y la RCIU. Siendo nuestra muestra 
no amplia, se sugiere ampliar la muestra de estudio, así como continuar con otras investigaciones sobre la relación del COMT Val/Met y la restricción de crecimiento intrauterino, de manera de aportar en el diagnóstico y manejo de esta preocupante complicación del feto y del recién nacido.

\section{REFERENCIAS BIBLIOGRÁFICAS}

1. Pacheco J, Oliveros M. Restricción en el crecimiento fetal intrauterino. En: Pacheco J. Ginecología Obstetricia y Reproducción. Lima, Perú: REP SAC. 2007:1380-98.

2. Pacheco J, Villacorta A, Del Carpio L, Velásquez E, Lozano E. Influencia de la preeclampsia/eclampsia en la gestante peruana y su recién nacido, 20062007. An Fac med. 2009;70 supl.1:S26.

3. Bernstein IM, Horbar JD, Badger GJ, Ohlsson A Golan A. Morbidity and mortality among very-lowbirth-weight neonates with intrauterine growth restriction. The Vermont Oxford Network. Am J Obste Gynecol. 2000;182(1 Pt 1):198-206.

4. Ticona M, Huanco. Factores de riesgo de la mortalidad perinatal en el Perú. Ginecol Obstet (Perú) 2003;49(4);227-36.

5. Delgado M, Muñoz A, Orejuela L, Sierra CH. Algunos factores de riesgo para mortalidad neonata en un hospital de III nivel, Popayán. Colomb Med. 2003;34:179-85.

6. Sata F, Yamada H, Susuki K, Saijo Y, Yamada T, Minakami H, Kishi R. Functional maternal catecholO-methyltransferase polymorphism and fetal growth restriction. Pharmacogenet Genomics. 2006;16:77581

7. Kramer MS. The epidemiology of adverse pregnancy outcomes: an overview. J Nutr. 2003;133:1592S1596s.

8. Vélez Gómez Maria del Pilar, Barros Fernando C, Echavarría Restrepo Luis Guillermo, Patricia. HAM. Prevalencia de bajo peso al nacer y factores maternos asociados: Unidad de Atención y Protección Materno Infantil de la Clínica Universitaria Bolivariana, Medellín, Colombia. Rev Colomb Obstet Ginecol. 2006;57:264-70.

9. Strauss RS. Adult functional outcome of those born small for gestational age: twenty-six-year follow-up of the 1970 British Birth Cohort. JAMA 2000;283(5):625-32.
10. Hack M, Schluchter M, Cartar L, Rahman M, Cuttler L, Borawski E. Growth of very low birth weight infants to age 20 years. Pediatrics. 2003;112(1 Pt 1):e30-8.

11. Law TL, Korte JE, Katikaneni LD, Wagner CL, Ebeling MD, Newman RB. Ultrasound assessment of intrauterine growth restriction: relationship to neonatal body composition. Am J Obstet Gynecol. 2011;205(3):255.e1-6.

12. Lee W, Balasubramaniam M, Deter RL, Hassan SS, Gotsch F, Kusanovic JP, Gonçalves LF, Romero R. Fetal growth parameters and birth weight: their relationship to neonatal body composition. Ultrasound Obstet Gynecol. 2009;33(4):441-6.

13. Pacheco J, Wagner P, Williams MA, Sánchez S. Enfermedades hipertensivas en la gestación. En: Pacheco J. Ginecologia, Obstetricia y Reproducción. $2^{a}$ Edición. Lima: REP SAC. 2007:1097-130.

14. Genetics Home Reference. COMT. Disponible en: http://ghr.nlm.nih.gov/gene/COMT. Obtenido el 17 de noviembre de 2011

15. Männistö PT, Kaakkola S. Catechol-O-methyltransferase (COMT): biochemistry, molecular biology, pharmacology, and clinical efficacy of the new selective COMT inhibitors. Pharmacol Rev. 1999;51(4):593-628.

16. Hill LD, York TP, Kusanovic JP, Gomez R, Eaves LJ, Romero R, Strauss JP III. Epistasis between COMT and MTHFR in maternal-fetal dyads increases risk for preeclampsia. PLoS One. 2011;6(1):e16681.

17. WMA Declaration of Helsinki - Ethical principles for research involving human subjects. Disponible en: http://www.wma.net/en/30publications/10policies/ b3/index.html. Obtenido el 17 de noviembre de 2011.

18. Zhang Z, Lindpaintner K, Che R, He Z, Wang P, Yang $P$, Feng $G, H e L$, Shi $Y$. The Val/Met functional polymorphism in COMT confers susceptibility to bipolar disorder: evidence from an association study and a meta-analysis. J Neural Transm. 2009;116(10):1193200.

19. Huerta D, Acosta O, Polo S, Martinez R, Oré R, Miranda C. Polimorfismo Val108/158Met en el gen dopaminérgico catecolo-metil transferasa (COMT) en una población mixta peruana y su importancia para los estudios neuropsiquiátricos. An Fac med. 2007;68(4):321-7.

20. Wen W, Cai Q, Shu XO, Cheng JR, Parl F, Pierce L, Gao YT, Zheng W. Cytochrome P450 1B1 and catechol-O-methyltransferase genetic polymorphisms and breast cancer risk in Chinese women: results from the Shanghai breast cancer study and a meta-analysis. Cancer Epidemiol Biomarkers Prev. 2005;14(2):329-35.
21. Zhao XM, Xie MQ, Yang DZ, Wang LA, Li SJ, Zhuang YY, Tang XL. [Polymorphism of catecholO-methyltransferase gene in relation to the risk of endometrial cancer]. Zhonghua Fu Chan Ke Za Zhi. 2007;42(2):116-9.

22. Osawa $Y$, Higashiyama T, Shimizu $Y$, Yarborough C. Multiple functions of aromatase and the active site structure; aromatase is the placental estrogen 2-hydroxylase. J Steroid Biochem Mol Biol. 1993;44(4-6):469-80.

23. Kanasaki K, Palmsten $K$, Sugimoto $H$, Ahmad $S$, Hamano Y, Xie L, Parry S, Augustin HG, Gattone VH, Folkman J, Strauss JF, Kalluri R. Deficiency in catechol-O-methyltransferase and 2-methoxyoestradiol is associated with pre-eclampsia. Nature. 2008 Jun 19;453(7198):1117-21.

24. Lim JH, Kim SY, Kim do J, Park SY, Han HW, Han JY, Lee SW, Yang JH, Ryu HM. Genetic polymorphism of catechol-O-methyltransferase and cytochrome P450c17 $\alpha$ in preeclampsia. Pharmacogenet Genomics. 2010;20(10):605-10.

25. Roten LT, Fenstad MH, Forsmo S, Johnson MP, Moses EK, Austgulen R, Skorpen F. A low COMT activity haplotype is associated with recurrent preeclampsia in a Norwegian population cohort (HUNT2). Mol Hum Reprod. 2011;17(7):439-46.

26. Shenoy V, Kanasaki K, Kalluri R. Pre-eclampsia: connecting angiogenic and metabolic pathways. Trends Endocrinol Metab. 2010;21(9):529-36.

27. Palmer K, Saglam B, Whitehead C, Stock O, Lappas $\mathrm{M}$, Tong S. Severe early-onset preeclampsia is not associated with a change in placental catechol Omethyltransferase (COMT) expression. Am J Pathol. 2011;178(6):2484-8.

28. Stanley JL, Andersson IJ, Poudel R, Rueda-Clausen CF, Sibley CP, Davidge ST, Baker PN. Sildenafi citrate rescues fetal growth in the catechol-O-methyl transferase knockout mouse model. Hypertension. 2012;59(5):1021-8.

Artículo recibido el 10 de setiembre de 2012 y aceptado para publicación el 28 de enero de 2013.

Financiamiento: Vicerrectorado de InvestigaciónConsejo Superior de Investigaciones, Universidad Nacional Mayor de San Marcos - Código del estudio: 110103201.

\section{Correspondencia:}

Dr. José Pacheco Romero

Correo electrónico: jpachecoperu@yahoo.com 